\title{
Study on Dose Rate on the Surface of Cask Packed with Activated Cut-off Pieces from Decommissioned Nuclear Power Plant
}

\author{
Kwang Soo Park', Hae Woong Kim', Hee Dong Sohn', Nam Kyun Kim², Chung Kyu Lee², Yun Lee², Ji Hoon Lee, \\ Young Hwan Hwang ${ }^{3}$, Mi Hyun Lee ${ }^{3}$, Dong Kyu Lee ${ }^{4}$, Duk Woon Jung ${ }^{4}$ \\ ${ }^{1}$ Nuclear Decommissioning Technology \& Business Development Team, Doosan Heavy Industries and Construction, Changwon, Korea; ${ }^{2} R A F$ Team, KEPCO \\ KPS, Naju, Korea; ${ }^{3}$ Radiation \& Environmental Laboratory, Korea Hydro \& Nuclear Power Co. Ltd., Daejeon, Korea; ${ }^{4}$ Nuclear Energy Team, KONES \\ Corporation, Seoul, Korea
}

\section{Technical Paper}

Received August 30, 2020

Revision October 15, 2020

Accepted October 30, 2020

Corresponding author: Kwang Soo Park

Doosan Heavy Industries and Construction, 555-1 Gwigok-dong, Seongsan-gu, Changwon 51711, Korea E-mail:kwangsoo.park@doosan.com

(iD https://orcid.org/0000-0002-0442-472X

\begin{abstract}
This is an open-access article distributed under the terms of the Creative Commons Attribution License (http://creativecommons.org/licenses/by-nc/4.0) which permits unrestricted use, distribution, and reproduction in any medium, provided the original work is properly cited.
\end{abstract}

Copyright $\odot 2020$ The Korean Association for Radiation Protection

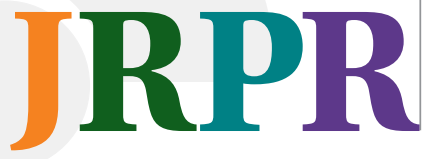

Background: Reactor pressure vessel (RV) with internals (RVI) are activated structures by neutron irradiation and volume contaminated wastes. Thus, to develop safe and optimized disposal plan for them at a disposal site, it is important to perform exact activation calculation and evaluate the dose rate on the surface of casks which contain cut-off pieces.

Materials and Methods: RV and RVI are subjected to neutron activation calculation via Monte Carlo methodology with MCNP6 and ORIGEN-S program-neutron flux, isotopic specific activity, and gamma spectrum calculation on each component of RV and RVI, and dose rate evaluation with MCNP6.

Results and Discussion: Through neutron activation analysis, dose rate is evaluated for the casks containing cut-off pieces produced from decommissioned RV and RVI. For RV cut-off ones, the highest value of dose rate on the surface of cask is $6.97 \times 10^{-1} \mathrm{mSv} / \mathrm{hr}$ and $2 \mathrm{~m}$ from it is $3.03 \times 10^{-2} \mathrm{mSv} / \mathrm{hr}$. For RVI cut-off ones, on the surface of it is $0.166 \times 10^{-1} \mathrm{mSv} / \mathrm{hr}$ and $2 \mathrm{~m}$ from it is $1.04 \times 10^{-1} \mathrm{mSv} / \mathrm{hr}$. Dose rates for various RV and RVI cut-off pieces distributed lower than the limit except the one of $2 \mathrm{~m}$ from the cask surface of RVI. It needs to adjust contents in cask which carries highly radioactive components in order to decrease thickness of cask.

Conclusion: Two types of casks are considered in this paper: box type for very-low-level waste (VLLW) as well as low-level waste (LLW) and cylinder type for intermediate-level waste (ILW). The results will contribute to the development of optimal loading plans for RV and RVI cut-off pieces during the decommissioning of nuclear power plant that can be used to prepare radioactive waste disposal plans for the different types of wastes-ILW, LLW, and VLLW.

Keywords: Monte Carlo, Cask, Drum, Reactor Pressure Vessel, Internal, Decommissioning

\section{Introduction}

When decommissioning a nuclear power plant, lots of metal wastes are produced and various studies have been carried out to characterize and to recycle them [1-3]. In South Korea, Kori Unit 1 is expected to be decommissioned and activation analysis for reactor pressure vessel (RV), reactor pressure vessel internal (RVI), and bio-shield has been carried out [4]. RV is composed of carbon steel and most RVI's are composed of stainless steel type 304 . The specific activities of RVI lie between $3.0 \times 10^{3} \mathrm{~Bq} / \mathrm{g}$ and 
$6.0 \times 10^{9} \mathrm{~Bq} / \mathrm{g}$ [4] depending on installation position in the core. In order to classify radioactive waste level and to make a plan for transportation and storage, the specific activity data is very important for RV and RVI components. Most irradiated RV and RVI are classified into very-low-level waste (VLLW), low-level waste (LLW), and intermediate-level waste (ILW) depending on the specific activity. After several years cooling period, the irradiated metal components shall be transported to the waste deposition site.

In order to transport RV and RVI components' cut-off pieces safely, it is necessary to be cut into proper sized pieces. The cutting pieces are designed based on the load weight, radioactive waste level, and cask size. Thus, the cut-off pieces become smaller in order to load more possible and to decrease number of casks. The cask shape is also important parameter. In this study, two kinds of casks are considered such as the box type and the cylinder type. An optimization analysis for loading radioactive wastes is carried out to decrease number of casks and to minimize load burden.

In this paper, an optimal loading is suggested for $\mathrm{RV}$ and RVI components based on the load balanced and shielding analysis. The radiation shielding is one of the important parameters to transport radioactive wastes. Therefore, the Monte Carlo simulation is carried out to estimate the surface dose rate of various cases of cut-off pieces loaded in two kinds of casks.

In Section 2, a brief description is provided on the cutting procedure and loading strategy. Section 3 provides activation analysis simulation results and loading conditions. In Section 4, shielding analyses are carried out for various casks for RV and RVI components.

Table 1. RV and RVI Component and Material

\begin{tabular}{lll}
\hline System & \multicolumn{1}{c}{ Component } & \multicolumn{1}{c}{ Material } \\
\hline $\begin{array}{c}\text { Reactor press } \\
\text { vessel (RV) }\end{array}$ & Reactor vessel & Carbon steel \\
& Reactor vessel head & Carbon steel \\
& Inlet/Outlet nozzle & Carbon steel \\
& ICl nozzle & Inconel \\
Reactor vessel & Former & Stainless steel type 304 \\
internal (RVI) & Baffle & Stainless steel type 304 \\
& Core barrel & Stainless steel type 304 \\
& Thermal shield & Stainless steel type 304 \\
& Upper core plate & Stainless steel type 304 \\
& Upper internal & Stainless steel type 304 \\
& Lower core plate & Stainless steel type 304 \\
& Lower internal & Stainless steel type 304 \\
\hline
\end{tabular}

$\mathrm{ICl}$, in-core instrumentation.

\section{Materials and Methods}

\section{Cutting Procedure and Loading Strategy}

Main materials of RV and RVI are stainless steel type 304, carbon steel (SA508 Grade 2 forged material) and Inconel as shown in Table 1. For the transportation of VLLW and LLW based on the activation calculation and cutting plan, boxtype cask will be used as depicted in Fig. 1. The specification of box-type cask is described in Table 2. The allowed maximum content weight is about 10 ton and it is specially designed to load in under water condition. Therefore, stillage is equipped for the safe loading in the water, too. The stillage material is carbon steel and other body components are composed of stainless steel type 304. The main reason of using box-type casks for VLLW and LLW is to optimize the packing condition in the cask, so it is possible to reduce the number of casks. The most important things for decommissioning nuclear power plant are not only safety but also economy, even though safety is much more important. After RV and RVI are dismantled safely and cut-off pieces are pro-
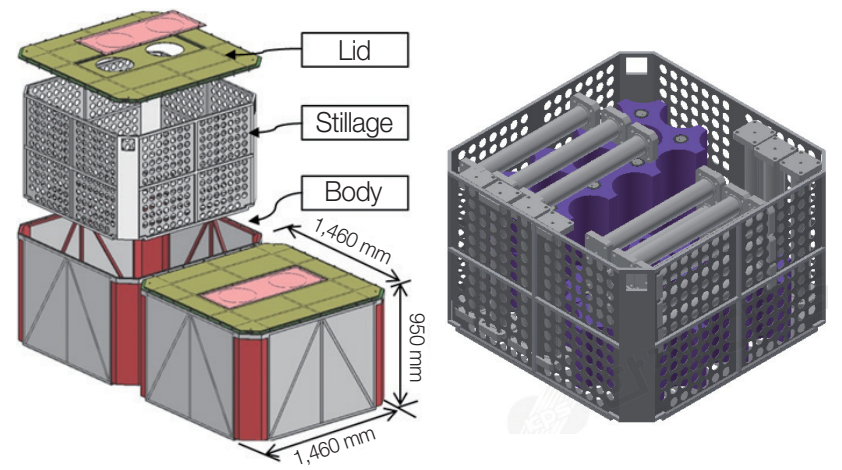

Fig. 1. Box-type cask for radioactive waste package.

Table 2. Specification of Box-Type Cask

\begin{tabular}{ll}
\hline Item & \multicolumn{1}{c}{ Parameter } \\
\hline Size (m) & $1.46 \times 1.46 \times 0.95$ (height) \\
Empty weight (ton) & 0.84 \\
Content weight (ton) & $\sim 5.5$ \\
Total weight including grouting (ton) & 9.4 \\
Thickness (cm) & 2.0 for RV \\
& 20.0 for RVI \\
Material & Stillage: carbon steel \\
& Body: stainless steel (stainless steel \\
& type 304) \\
Grouting & After water drain \\
Stillage & For underwater loading \\
Salvage & Specified shielding bell salvage \\
\hline
\end{tabular}

$\mathrm{RVI}$, reactor vessel internal. 
duced, it is necessary to consider economy as achievable as possible in the second place. It means that radioactive waste disposal expense should be reduced with optimized cask packing design through dose rate evaluation on the surface of it. If the certified $200 \mathrm{~L}$ drum is used for VLLW and LLW, the estimated number of drum is about 484 . However, only 48 box-type casks are required in order to store and pack the same contents. It is achieved both reduction of transportation burden and economic benefit considering movement from decommissioning site to disposal place. Fig. 2 shows loading examples when using the typical $200 \mathrm{~L}$ drum. It is found that lots of vacant area and inefficient regions exist in each drum. However, there is little vacant area in box-type cask and it is more efficient to store wastes.

For the purpose of ILW storage, cylinder-type cask is taken

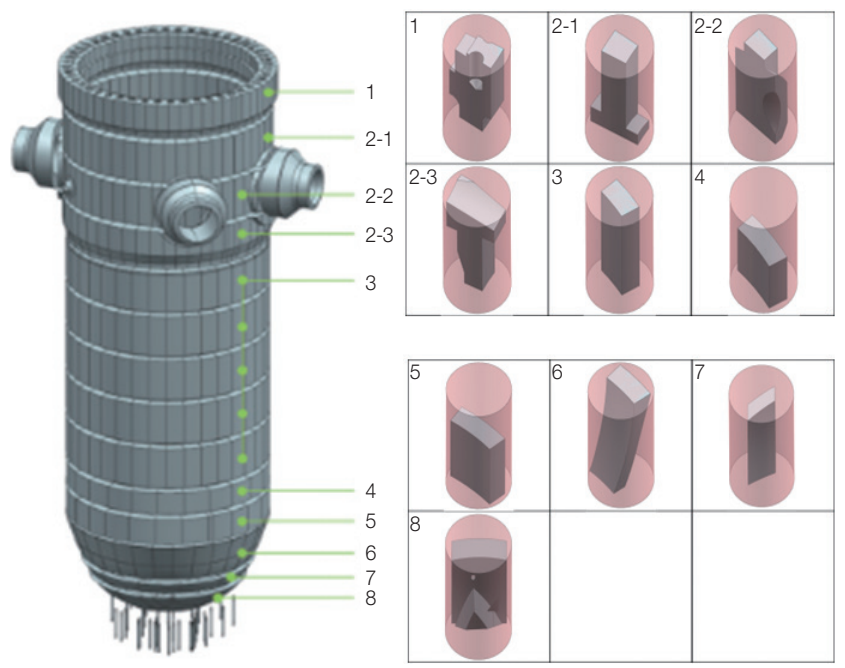

Fig. 2. Loading example in $200 \mathrm{~L}$ drum for reactor pressure vessel.

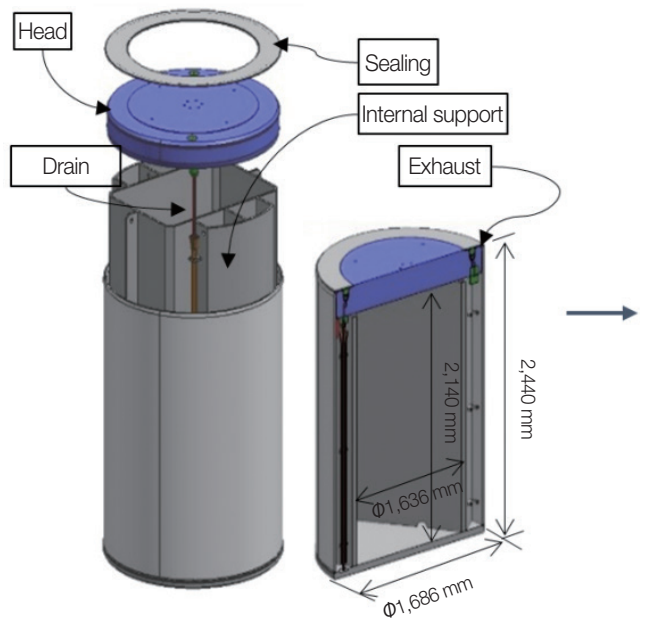

into consideration as depicted in Fig. 3. The specification of the cylinder-type cask is described in Table 3. Allowed maximum content weight is about 25 ton. Cask material is stainless steel type 304 which is good for both shielding capability and structural maintenance. Outer body of cask material is made of concrete whose thickness is about $41 \mathrm{~cm}$.

Two main strategies for packing plan are developed as follows. The first consideration item is to store as many cut-off pieces as possible in the cask. Thus maximum volume and weight become the main dependent parameters to be considered. The second one is to meet the radiation shielding requirement on the surface of it for safety. So, in order to store maximum loading contents, the size of each cut-off piece should be produced as much smaller, otherwise, it requires more number of casks and it becomes uneconomical. Thus, optimized cutting plan applying activation evaluation result and cask size should be prepared, and loading contents should meet satisfaction of dose rate on the surface of casks.

When RV is planned to be dismantled, several small cutoff pieces are produced due to complex and curved shape

Table 3. Specification of Cylinder-Type Cask

\begin{tabular}{ll}
\hline Item & \multicolumn{1}{c}{ Parameter } \\
\hline Outer size $(\mathrm{m})$ & $1.686 \times 2.44$ (diameter $\times$ height) \\
Inner size $(\mathrm{m})$ & $1.636 \times 2.14$ (diameter $\times$ height) \\
Empty weight (ton) & 10.3 \\
Content weight (ton) & $\sim 15.0$ \\
Total weight including grouting (ton) & 25.3 \\
Thickness of outer concrete $(\mathrm{cm})$ & 41.0 \\
Material & Stainless steel (stainless steel type \\
& $304)$ \\
Equipment & Head drain/Dry facility \\
\hline
\end{tabular}
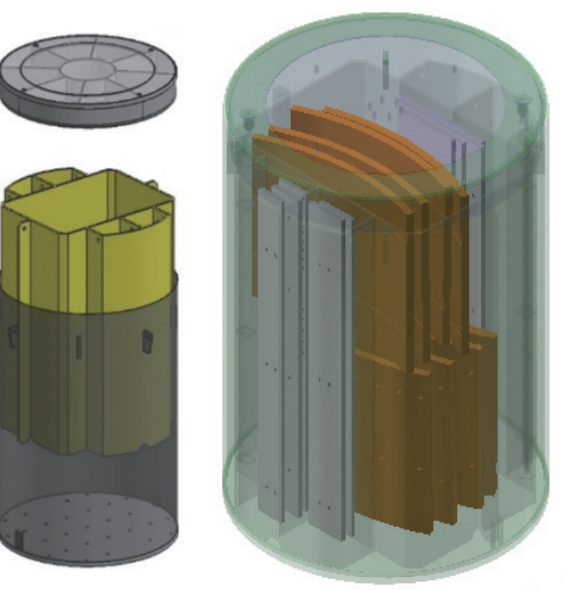

Fig. 3. Cylinder-type cask for radioactive waste storage. 
considering loading capacity in cask. As many small cut-off pieces as produced, cutting procedure also requires more time and man-power, so, cutting strategy should be prepared for all aspects. Fig. 4 shows an example of loading configurations for RV cut-off pieces in the box-type cask. As expected, various shapes of RV curvature play main role of planning cutting procedure. The thicknesses of box-type cask are designed as $2 \mathrm{~cm}$ for both RV and $20 \mathrm{~cm}$ for RVI components caused by the difference of radioactive waste level.

\section{Results and Discussion}

\section{Dose Rate Evaluation of Cask for RV and RVI Cut-off Pieces}

MCNP6 [5] code is used to calculate the neutron flux distribution during irradiation period and ORIGEN-S [6] code is also used to evaluate isotopic inventories. From the analysis of 40 years irradiation, the total decay gamma intensity and spectrum are obtained by including various impurities for stainless steel type 304 and carbon steel [7]. In case of carbon steel in RV central region, the specific activities of various isotopes are tabulated in Table 4. Fig. 5 depicts the gamma spectrum after 10 year cooling for $1 \mathrm{~g}$ of carbon steel. Table 5 provides activation analysis results for stainless steel type 304 in RVI component. Fig. 6 also shows the gamma spectrum after 10-year cooling for $1 \mathrm{~g}$ of stainless steel type 304 .

The contents of RV and RVI components are classified based on the total activity for satisfaction of limit surface dose rate. Table 6 summarizes RV components. Lots of components are included such as RV main body, RV bottom head, a bottom-mounted instrumentation (BMI) nozzle, inlet and outlet nozzle, and insulation. Table 7 provides RVI

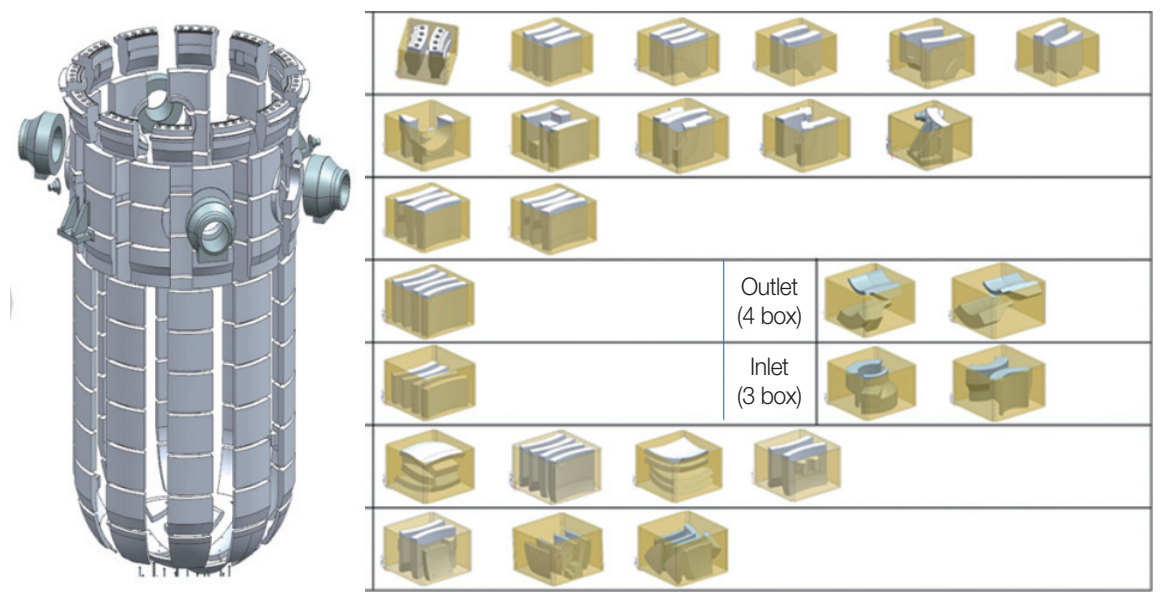

Fig. 4. An example of optimal loading for the box-type cask for reactor pressure vessel components.

Table 4. Isotopic Specific Activities for RV for Various Cooling Time

\begin{tabular}{lccccc}
\hline & \multicolumn{5}{c}{ Specific activity $(\mathrm{Bq} / \mathrm{g})$} \\
\cline { 2 - 6 } Isotope & Discharge & $\begin{array}{r}30 \text {-day } \\
\text { cooling }\end{array}$ & $\begin{array}{c}\text { 2-year } \\
\text { cooling }\end{array}$ & $\begin{array}{c}5 \text {-year } \\
\text { cooling }\end{array}$ & $\begin{array}{c}10 \text {-year } \\
\text { cooling }\end{array}$ \\
\hline${ }^{3} \mathrm{H}$ & $1.05 \times 10^{3}$ & $1.05 \times 10^{3}$ & $9.41 \times 10^{2}$ & $7.95 \times 10^{2}$ & $6.00 \times 10^{2}$ \\
${ }^{14} \mathrm{C}$ & $2.28 \times 10^{1}$ & $2.28 \times 10^{1}$ & $2.28 \times 10^{1}$ & $2.28 \times 10^{1}$ & $2.28 \times 10^{1}$ \\
${ }^{60} \mathrm{Co}$ & $7.10 \times 10^{4}$ & $7.01 \times 10^{4}$ & $5.46 \times 10^{4}$ & $3.68 \times 10^{4}$ & $1.91 \times 10^{4}$ \\
${ }^{59} \mathrm{Ni}$ & $4.77 \times 10^{3}$ & $4.77 \times 10^{3}$ & $4.77 \times 10^{3}$ & $4.77 \times 10^{3}$ & $4.77 \times 10^{3}$ \\
${ }^{63} \mathrm{Ni}$ & $5.01 \times 10^{5}$ & $5.01 \times 10^{5}$ & $4.95 \times 10^{5}$ & $4.84 \times 10^{5}$ & $4.68 \times 10^{5}$ \\
${ }^{90} \mathrm{Sr}$ & $6.91 \times 10^{-10}$ & $6.89 \times 10^{-10}$ & $6.58 \times 10^{-10}$ & $6.11 \times 10^{-10}$ & $5.40 \times 10^{-10}$ \\
${ }^{94} \mathrm{Nb}$ & $6.01 \times 10^{-1}$ & $6.01 \times 10^{-1}$ & $6.01 \times 10^{-1}$ & $6.01 \times 10^{-1}$ & $6.01 \times 10^{-1}$ \\
${ }^{99} \mathrm{TC}$ & $2.48 \times 10^{-4}$ & $2.48 \times 10^{-4}$ & $2.48 \times 10^{-4}$ & $2.48 \times 10^{-4}$ & $2.48 \times 10^{-4}$ \\
${ }^{129} \mathrm{I}$ & $2.64 \times 10^{-17}$ & $2.64 \times 10^{-17}$ & $2.64 \times 10^{-17}$ & $2.64 \times 10^{-17}$ & $2.64 \times 10^{-17}$ \\
${ }^{137} \mathrm{Cs}$ & $3.78 \times 10^{-11}$ & $3.77 \times 10^{-11}$ & $3.61 \times 10^{-11}$ & $3.37 \times 10^{-11}$ & $3.00 \times 10^{-11}$ \\
${ }^{T} \mathrm{Total}$ & $9.42 \times 10^{7}$ & $9.09 \times 10^{7}$ & $5.24 \times 10^{7}$ & $2.41 \times 10^{7}$ & $7.07 \times 10^{6}$ \\
\hline
\end{tabular}

$\mathrm{RV}$, reactor pressure vessel.

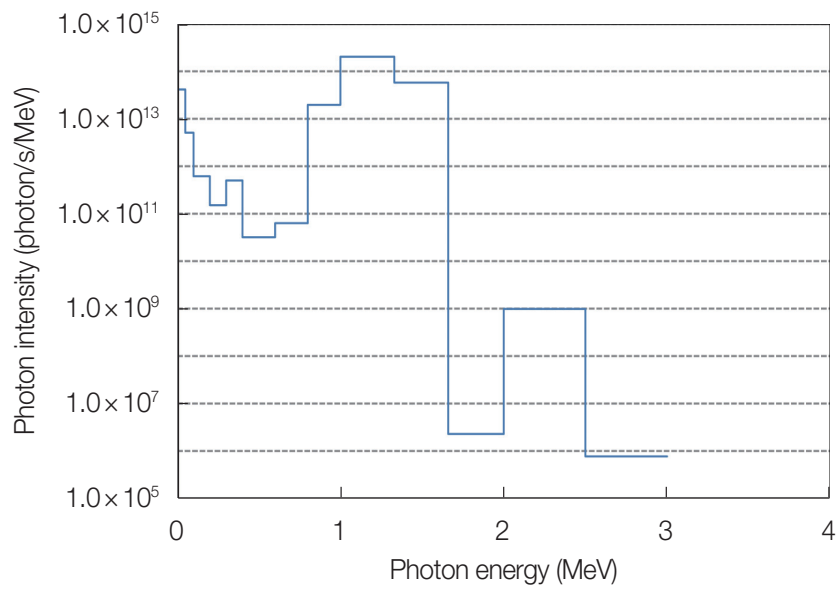

Fig. 5. Gamma spectrum for reactor pressure vessel for 10-year cooling. 


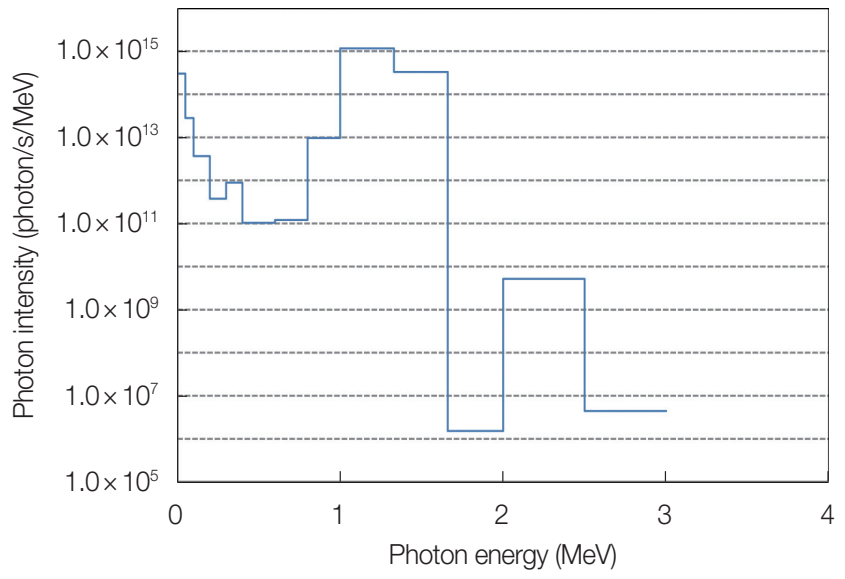

Fig. 6. Gamma spectrum for reactor pressure vessel internal for 10year cooling.

Table 5. Isotopic Specific Activities for RVI for Various Cooling Time

\begin{tabular}{lccccc}
\hline & \multicolumn{5}{c}{ Specific activity $(\mathrm{Bq} / \mathrm{g})$} \\
\cline { 2 - 6 } Isotope & Discharge & $\begin{array}{c}30 \text {-day } \\
\text { cooling }\end{array}$ & $\begin{array}{c}\text { 2-year } \\
\text { cooling }\end{array}$ & $\begin{array}{c}5 \text {-year } \\
\text { cooling }\end{array}$ & $\begin{array}{c}10 \text {-year } \\
\text { cooling }\end{array}$ \\
\hline${ }^{63} \mathrm{Ni}$ & $5.29 \times 10^{7}$ & $5.29 \times 10^{7}$ & $5.22 \times 10^{7}$ & $5.11 \times 10^{7}$ & $4.94 \times 10^{7}$ \\
${ }^{60} \mathrm{Co}$ & $1.00 \times 10^{7}$ & $9.91 \times 10^{6}$ & $7.72 \times 10^{6}$ & $5.20 \times 10^{6}$ & $2.69 \times 10^{6}$ \\
${ }^{59} \mathrm{Ni}$ & $4.86 \times 10^{5}$ & $4.86 \times 10^{5}$ & $4.86 \times 10^{5}$ & $4.86 \times 10^{5}$ & $4.86 \times 10^{5}$ \\
${ }^{3} \mathrm{H}$ & $4.40 \times 10^{3}$ & $4.38 \times 10^{3}$ & $3.94 \times 10^{3}$ & $3.32 \times 10^{3}$ & $2.51 \times 10^{3}$ \\
${ }^{14} \mathrm{C}$ & $8.59 \times 10^{2}$ & $8.59 \times 10^{2}$ & $8.59 \times 10^{2}$ & $8.59 \times 10^{2}$ & $8.58 \times 10^{2}$ \\
${ }^{94} \mathrm{Nb}$ & $1.96 \times 10^{1}$ & $1.96 \times 10^{1}$ & $1.96 \times 10^{1}$ & $1.96 \times 10^{1}$ & $1.96 \times 10^{1}$ \\
${ }^{99} \mathrm{TC}$ & 7.65 & 7.65 & 7.65 & 7.65 & 7.65 \\
${ }^{137} \mathrm{Cs}$ & $1.56 \times 10^{-4}$ & $1.56 \times 10^{-4}$ & $1.49 \times 10^{-4}$ & $1.39 \times 10^{-4}$ & $1.24 \times 10^{-4}$ \\
${ }^{90} \mathrm{Sr}$ & $2.69 \times 10^{-6}$ & $2.69 \times 10^{-6}$ & $2.57 \times 10^{-6}$ & $2.38 \times 10^{-6}$ & $2.11 \times 10^{-6}$ \\
${ }^{129}$ & $2.46 \times 10^{-13}$ & $2.46 \times 10^{-13}$ & $2.46 \times 10^{-13}$ & $2.46 \times 10^{-13}$ & $2.46 \times 10^{-13}$ \\
Total & $1.41 \times 10^{9}$ & $6.89 \times 10^{8}$ & $3.85 \times 10^{8}$ & $2.03 \times 10^{8}$ & $9.32 \times 10^{7}$ \\
\hline
\end{tabular}

$\mathrm{RV}$, reactor pressure vessel internal.
Table 6. Cask Information for RV Components

\begin{tabular}{|c|c|c|c|c|c|}
\hline Contents & $\begin{array}{l}\text { Cask } \\
\text { ID }\end{array}$ & $\begin{array}{l}\text { Cask } \\
\text { type }\end{array}$ & $\begin{array}{l}\text { Fixed } \\
\text { weight } \\
\text { (ton) }\end{array}$ & $\begin{array}{c}\text { Total } \\
\text { activity } \\
\text { (MBq) }\end{array}$ & $\begin{array}{c}\text { Photon } \\
\text { intensity } \\
\text { (photons/s) }\end{array}$ \\
\hline \multirow[t]{17}{*}{ RV main body } & RV-01 & Box & 6.89 & $4.97 \times 10^{4}$ & $2.88 \times 10^{9}$ \\
\hline & RV-02 & Box & 5.83 & $5.28 \times 10^{4}$ & $3.06 \times 10^{9}$ \\
\hline & RV-03 & Box & 4.94 & $5.08 \times 10^{4}$ & $2.94 \times 10^{9}$ \\
\hline & RV-04 & Box & 5.87 & $3.54 \times 10^{4}$ & $2.05 \times 10^{9}$ \\
\hline & RV-05 & Box & 6.01 & $3.73 \times 10^{4}$ & $2.16 \times 10^{9}$ \\
\hline & RV-06 & Box & 6.05 & $3.79 \times 10^{4}$ & $2.19 \times 10^{9}$ \\
\hline & RV-07 & Box & 5.33 & $2.78 \times 10^{4}$ & $1.61 \times 10^{9}$ \\
\hline & RV-08 & Box & 7.15 & $5.33 \times 10^{4}$ & $3.09 \times 10^{9}$ \\
\hline & RV-09 & Box & 6.84 & $4.91 \times 10^{4}$ & $2.84 \times 10^{9}$ \\
\hline & $\mathrm{RV}-10$ & Box & 5.95 & $3.66 \times 10^{4}$ & $2.12 \times 10^{9}$ \\
\hline & $\mathrm{RV}-11$ & Box & 5.73 & $3.34 \times 10^{4}$ & $1.93 \times 10^{9}$ \\
\hline & $\mathrm{RV}-12$ & Box & 5.73 & $3.34 \times 10^{4}$ & $1.93 \times 10^{9}$ \\
\hline & RV-13 & Box & 7.15 & $5.33 \times 10^{4}$ & $3.09 \times 10^{9}$ \\
\hline & RV-14 & Box & 7.14 & $5.33 \times 10^{4}$ & $3.09 \times 10^{9}$ \\
\hline & $R V-15$ & Box & 7.05 & $1.68 \times 10^{6}$ & $9.73 \times 10^{10}$ \\
\hline & $R V-16$ & Box & 7.05 & $1.68 \times 10^{6}$ & $9.73 \times 10^{10}$ \\
\hline & $\mathrm{RV}-17$ & Box & 5.88 & $1.15 \times 10^{6}$ & $6.66 \times 10^{10}$ \\
\hline \multirow{2}{*}{$\begin{array}{l}\text { RV main body/ } \\
\text { RV bottom head }\end{array}$} & RV-18 & Box & 5.84 & $6.81 \times 10^{5}$ & $3.94 \times 10^{10}$ \\
\hline & RV-19 & Box & 6.94 & $1.25 \times 10^{6}$ & $7.24 \times 10^{10}$ \\
\hline RV bottom head & RV-20 & Box & 5.72 & $3.19 \times 10^{2}$ & $1.85 \times 10^{7}$ \\
\hline \multirow{3}{*}{$\begin{array}{l}\text { RV main body/ } \\
\text { RV bottom head }\end{array}$} & $\mathrm{RV}-21$ & Box & 5.78 & $6.75 \times 10^{5}$ & $3.91 \times 10^{10}$ \\
\hline & RV-22 & Box & 5.78 & $6.75 \times 10^{5}$ & $3.91 \times 10^{10}$ \\
\hline & RV-23 & Box & 5.44 & $7.19 \times 10^{5}$ & $4.16 \times 10^{10}$ \\
\hline $\begin{array}{l}\text { RV bottom head/ } \\
\text { BMI Nozzle }\end{array}$ & RV-24 & Box & 4.93 & $3.64 \times 10^{4}$ & $2.11 \times 10^{9}$ \\
\hline RV bottom head & RV-25 & Box & 4.90 & $2.07 \times 10^{2}$ & $1.20 \times 10^{7}$ \\
\hline \multirow[t]{2}{*}{ Inlet } & RV-26 & Box & 4.98 & $7.70 \times 10^{3}$ & $4.46 \times 10^{8}$ \\
\hline & RV-27 & Box & 4.90 & $7.28 \times 10^{3}$ & $4.22 \times 10^{8}$ \\
\hline \multirow[t]{2}{*}{ Outlet } & RV-28 & Box & 4.82 & $6.93 \times 10^{3}$ & $4.01 \times 10^{8}$ \\
\hline & RV-29 & Box & 4.64 & $6.07 \times 10^{3}$ & $3.51 \times 10^{8}$ \\
\hline Insulation (bottom head) & RV-30 & Box & 5.14 & $2.03 \times 10^{3}$ & $1.18 \times 10^{8}$ \\
\hline Insulation (active fuel) & RV-31 & Box & 4.12 & $1.442 \times 10^{4}$ & $8.35 \times 10^{8}$ \\
\hline
\end{tabular}

$\mathrm{RV}$, reactor pressure vessel; BMl, bottom-mounted instrumentation.

Table 7. Cask Information for RVI Components

\begin{tabular}{lllccc}
\hline Contents & Cask ID & Cask type & Fixed weight (ton) & Total activity (MBq) & Photon intensity (photons/s) \\
\hline Thermal shield & RVI-01 & Cylinder & 13.2 & $7.64 \times 10^{8}$ & $4.61 \times 10^{13}$ \\
Thermal shield & RVI-02 & Cylinder & 9.9 & $5.96 \times 10^{8}$ & $4.15 \times 10^{14}$ \\
Baffle & RVI-02 & Cylinder & 1.2 & $3.13 \times 10^{9}$ & $4.15 \times 10^{14}$ \\
Former & RVI-02 & Cylinder & 1.4 & $3.15 \times 10^{9}$ & $4.15 \times 10^{14}$ \\
Thermal shield & RVI-03 & Cylinder & 14.1 & $7.95 \times 10^{8}$ & $4.80 \times 10^{13}$ \\
Baffle & RVI-04 & Cylinder & 3.8 & $1.00 \times 10^{10}$ & $7.94 \times 10^{14}$ \\
Former & RVI-04 & Cylinder & 1.4 & $3.15 \times 10^{9}$ & $7.94 \times 10^{14}$ \\
Core barrel & RVI-05 & Cylinder & 3.5 & $8.28 \times 10^{8}$ & $5.00 \times 10^{13}$ \\
Core barrel & RVI-06 & Cylinder & 5.9 & $1.40 \times 10^{9}$ & $8.45 \times 10^{13}$ \\
Upper core plate & RVI-07 & Box & 1.5 & $2.74 \times 10^{8}$ & $5.38 \times 10^{14}$ \\
Baffle & RVI-07 & Box & 3.0 & $7.94 \times 10^{9}$ & $5.38 \times 10^{14}$ \\
Guide tube & RVI-07 & Box & 1.9 & $3.45 \times 10^{8}$ & $5.38 \times 10^{14}$ \\
Support column (L) & RVI-07 & Box & 0.9 & $1.56 \times 10^{8}$ & $5.38 \times 10^{14}$ \\
Support column (S) & RVI-07 & Box & 0.6 & $1.00 \times 10^{8}$ & $5.38 \times 10^{14}$ \\
\hline
\end{tabular}


Table 7. Continued

\begin{tabular}{|c|c|c|c|c|c|}
\hline Contents & Cask ID & Cask type & Fixed weight (ton) & Total activity (MBq) & Photon intensity (photons/s) \\
\hline Mixing device stop & RVI-07 & Box & 0.5 & $9.74 \times 10^{7}$ & $5.38 \times 10^{14}$ \\
\hline Orifice plate (UCP) & RVI-07 & Box & 0.05 & $8.74 \times 10^{6}$ & $5.38 \times 10^{14}$ \\
\hline Core barrel & RVI-08 & Box & 3.5 & $8.22 \times 10^{8}$ & $2.30 \times 10^{14}$ \\
\hline Baffle & RVI-08 & Box & 1.5 & $2.99 \times 10^{9}$ & $2.30 \times 10^{14}$ \\
\hline Lower core plate & RVI-09 & Box & 0.3 & $3.28 \times 10^{6}$ & $2.03 \times 10^{11}$ \\
\hline Core support column & RVI-09 & Box & 1.1 & $2.99 \times 10^{4}$ & $2.03 \times 10^{11}$ \\
\hline Core support forging & RVI-09 & Box & 2.1 & $5.71 \times 10^{4}$ & $2.03 \times 10^{11}$ \\
\hline Core flange & RVI-10 & Box & 2.5 & $3.17 \times 10^{6}$ & $1.91 \times 10^{11}$ \\
\hline Core flange & RVI-11 & Box & 2.9 & $3.56 \times 10^{6}$ & $2.18 \times 10^{11}$ \\
\hline Mixing device stop & RVI-11 & Box & 0.5 & $4.06 \times 10^{4}$ & $2.18 \times 10^{11}$ \\
\hline Orifice plate & RVI-11 & Box & 0.05 & $6.05 \times 10^{3}$ & $2.18 \times 10^{11}$ \\
\hline Core flange & RVI-12 & Box & 2.9 & $8.64 \times 10^{3}$ & $1.89 \times 10^{11}$ \\
\hline Support column (L) & RVI-12 & Box & 0.9 & $3.12 \times 10^{6}$ & $1.89 \times 10^{11}$ \\
\hline Support column (S) & RVI-12 & Box & 0.2 & $6.80 \times 10^{1}$ & $1.89 \times 10^{11}$ \\
\hline Core supporting forging & RVI-13 & Box & 2.1 & $5.61 \times 10^{4}$ & $6.65 \times 10^{9}$ \\
\hline Butt instrument column & RVI-13 & Box & 0.8 & $2.05 \times 10^{4}$ & $6.65 \times 10^{9}$ \\
\hline Cruciform column & RVI-13 & Box & 0.2 & $4.46 \times 10^{3}$ & $6.65 \times 10^{9}$ \\
\hline Lowe core plate & RVI-13 & Box & 0.3 & $2.91 \times 10^{4}$ & $6.65 \times 10^{9}$ \\
\hline Core supporting forging & RVI-14 & Box & 1.5 & $4.06 \times 10^{4}$ & $5.76 \times 10^{9}$ \\
\hline Butt instrument column & RVI-14 & Box & 0.8 & $2.05 \times 10^{4}$ & $5.76 \times 10^{9}$ \\
\hline Lower core plate & RVI-14 & Box & 0.3 & $3.43 \times 10^{4}$ & $5.76 \times 10^{9}$ \\
\hline Core supporting forging & RVI-15 & Box & 1.5 & $4.06 \times 10^{4}$ & $3.77 \times 10^{11}$ \\
\hline Cruciform column & RVI-15 & Box & 0.2 & $6.05 \times 10^{3}$ & $3.77 \times 10^{11}$ \\
\hline Tie plate & RVI-15 & Box & 0.3 & $8.64 \times 10^{3}$ & $3.77 \times 10^{11}$ \\
\hline Lower core plate & RVI-15 & Box & 0.3 & $3.10 \times 10^{6}$ & $3.77 \times 10^{11}$ \\
\hline Guide tube & RVI-15 & Box & 0.3 & $3.10 \times 10^{6}$ & $3.77 \times 10^{11}$ \\
\hline Tie plate & RVI-16 & Box & 0.7 & $1.95 \times 10^{4}$ & $4.27 \times 10^{12}$ \\
\hline Cruciform column & RVI-16 & Box & 0.3 & $7.70 \times 10^{3}$ & $4.27 \times 10^{12}$ \\
\hline Lower core plate & RVI-16 & Box & 0.3 & $2.79 \times 10^{6}$ & $4.27 \times 10^{12}$ \\
\hline Guide tube & RVI-16 & Box & 0.7 & $6.80 \times 10^{7}$ & $4.27 \times 10^{12}$ \\
\hline Core supporting forging & RVI-17 & Box & 2.2 & $5.90 \times 10^{4}$ & $2.01 \times 10^{11}$ \\
\hline Cruciform column & RVI-17 & Box & 0.3 & $8.91 \times 10^{3}$ & $2.01 \times 10^{11}$ \\
\hline Energy absorber & RVI-17 & Box & 0.4 & $1.12 \times 10^{4}$ & $2.01 \times 10^{11}$ \\
\hline Lower core plate & RVI-17 & Box & 0.3 & $3.25 \times 10^{6}$ & $2.01 \times 10^{11}$ \\
\hline Guide tube & RVI-17 & Box & 0.5 & $5.10 \times 10^{1}$ & $2.01 \times 10^{11}$ \\
\hline Extension nozzle & RVI-18 & Box & 0.1 & $3.24 \times 10^{3}$ & $4.50 \times 10^{11}$ \\
\hline Butt instrument column & RVI-18 & Box & 0.2 & $5.67 \times 10^{3}$ & $4.50 \times 10^{11}$ \\
\hline Offset column instrument & RVI-18 & Box & 0.01 & $5.67 \times 10^{3}$ & $4.50 \times 10^{11}$ \\
\hline Core flange & RVI-18 & Box & 3.1 & $4.02 \times 10^{6}$ & $4.50 \times 10^{11}$ \\
\hline Support column & RVI-18 & Box & 0.2 & $1.81 \times 10^{1}$ & $4.50 \times 10^{11}$ \\
\hline Core flange & RVI-19 & Box & 3.4 & $4.41 \times 10^{6}$ & $2.66 \times 10^{11}$ \\
\hline Support column (S) & RVI-19 & Box & 1.8 & $1.81 \times 10^{1}$ & $2.66 \times 10^{11}$ \\
\hline Deep beam & RVI-19 & Box & 0.08 & 8.22 & $2.66 \times 10^{11}$ \\
\hline
\end{tabular}

$\mathrm{RVI}$, reactor pressure vessel internal.

components. One cask may contain several components based on the weight and total activity. Fig. 7 shows some typical casks which contain various RV and RVI components.

In order to transport safely RV and RVI cut-off pieces by using box and cylinder-type casks, the limit of surface dose rates are specified based on the Korean Nuclear Safety Law as follows:
- Surface of cask: $10 \mathrm{mSv} / \mathrm{hr}$

$-2 \mathrm{~m}$ from surface: $0.1 \mathrm{mSv} / \mathrm{hr}$

The box-type cask for RV components is modeled by MCNP6 in order to estimate dose rate. The model is shown in Fig. 8. Total particle simulation number is $1.0 \times 10^{7}$ in order to have sufficiently high reliability and the International Commission on Radiological Protection Publication 21 (ICRP-21) 

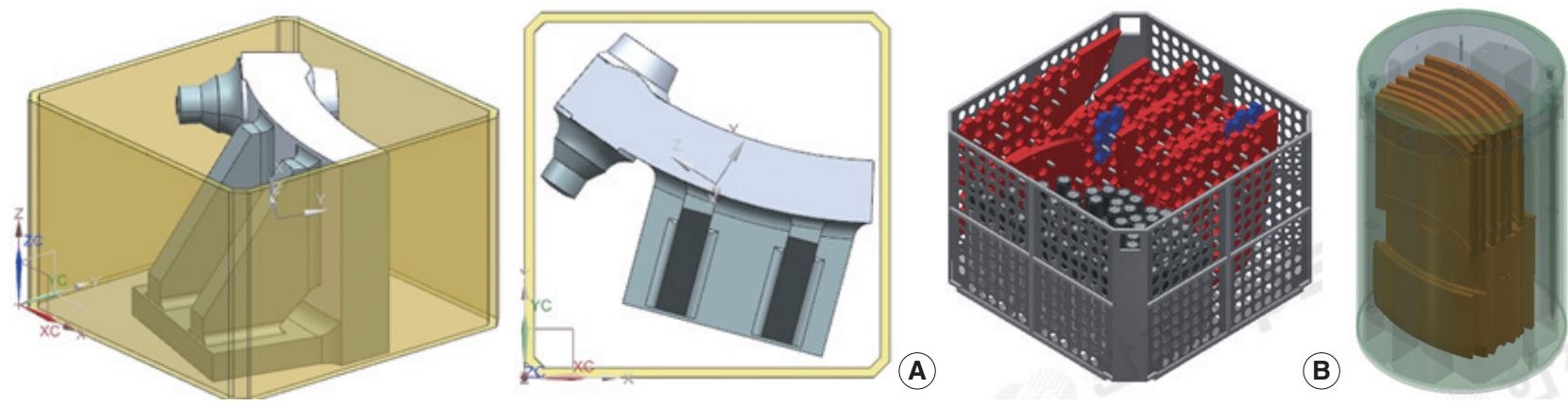

(C)

Fig. 7. Several examples of cask loading configurations: (A) reactor pressure vessel (upper shell), (B) reactor pressure vessel internal (lower core plate), and (C) reactor pressure vessel internal (core barrel).
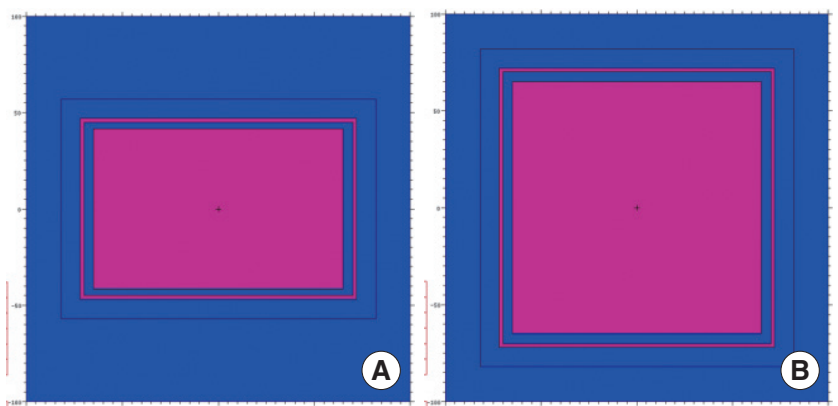

Fig. 8. Box-type cask model for shielding simulation: (A) horizontal view and $(B)$ vertical view.

Table 8. ICRP-21 Dose Conversion Factor

\begin{tabular}{lcc}
\hline $\begin{array}{l}\text { Lower energy } \\
(\mathrm{MeV})\end{array}$ & $\begin{array}{c}\text { Upper energy } \\
(\mathrm{MeV})\end{array}$ & $\begin{array}{c}\text { Conversion factor } \\
(\mathrm{mSv} / \mathrm{hr}) /(\text { photon/cm } / \mathrm{s})\end{array}$ \\
\hline $1.00 \times 10^{-2}$ & $5.00 \times 10^{-2}$ & $2.37 \times 10^{-2}$ \\
$5.00 \times 10^{-2}$ & $1.00 \times 10^{-1}$ & $2.76 \times 10^{-3}$ \\
$1.00 \times 10^{-1}$ & $2.00 \times 10^{-1}$ & $7.20 \times 10^{-4}$ \\
$2.00 \times 10^{-1}$ & $3.00 \times 10^{-1}$ & $7.40 \times 10^{-5}$ \\
$3.00 \times 10^{-1}$ & $4.00 \times 10^{-1}$ & $1.74 \times 10^{-4}$ \\
$4.00 \times 10^{-1}$ & $6.00 \times 10^{-1}$ & $4.07 \times 10^{-5}$ \\
$6.00 \times 10^{-1}$ & $8.00 \times 10^{-1}$ & $4.74 \times 10^{-5}$ \\
$8.00 \times 10^{-1}$ & 1.00 & $3.78 \times 10^{-3}$ \\
1.00 & 1.33 & $7.55 \times 10^{-1}$ \\
1.33 & 1.66 & $2.13 \times 10^{-1}$ \\
1.66 & 2.00 & $1.02 \times 10^{-9}$ \\
2.00 & 2.50 & $5.10 \times 10^{-6}$ \\
2.50 & 3.00 & $4.36 \times 10^{-9}$ \\
\hline
\end{tabular}

ICRP-21, International Commission on Radiological Protection Publication 21.

dose conversion factor is used as given in Table 8. When photon intensity of $2.8 \times 10^{9}$ photons/s/MeV is assumed for RV component, the surface dose rates are obtained from MCNP calculation using surface tally (F2) as given in Table 9. At the surface and $2 \mathrm{~m}$ from the box-type cask, $2.0 \times 10^{-2} \mathrm{mSv} / \mathrm{hr}$ and $8.7 \times 10^{-4} \mathrm{mSv} / \mathrm{hr}$ are obtained, respectively, which satisfy
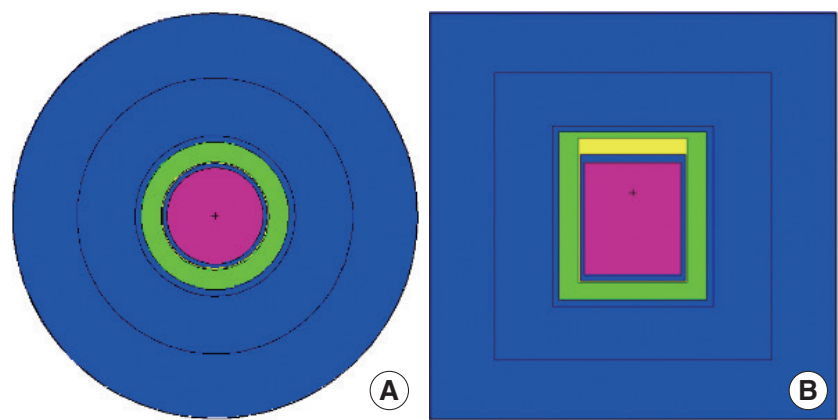

Fig. 9. Cylinder-type cask model for shielding calculation: (A) horizontal view and $(\mathrm{B})$ vertical view.

Table 9. Dose Rate at Various Positions for Box-Type Cask for RV Components

\begin{tabular}{lcc}
\hline & Dose rate $(\mathrm{mSv} / \mathrm{hr})$ & Relative error $\left.^{2}\right)$ \\
\hline Surface & $2.00 \times 10^{-2}$ & 0.0042 \\
$10 \mathrm{~cm}$ & $1.37 \times 10^{-2}$ & 0.0041 \\
$1 \mathrm{~m}$ & $2.33 \times 10^{-3}$ & 0.0038 \\
$2 \mathrm{~m}$ & $8.70 \times 10^{-4}$ & 0.0037 \\
\hline
\end{tabular}

$\mathrm{RV}$, reactor pressure vessel.

a) Relative error $=\frac{\text { Standard deviation }}{\text { Mean }}$.

Table 10. Dose Rate at Various Positions for the Cylinder-Type Cask for RVI Components

\begin{tabular}{lcc}
\hline & Dose rate $(\mathrm{mSv} / \mathrm{hr})$ & Relative error ${ }^{\mathrm{a})}$ \\
\hline Surface & $1.42 \times 10^{-3}$ & 0.1105 \\
$10 \mathrm{~cm}$ & $1.15 \times 10^{-3}$ & 0.1097 \\
$1 \mathrm{~m}$ & $3.92 \times 10^{-4}$ & 0.1111 \\
$2 \mathrm{~m}$ & $1.82 \times 10^{-4}$ & 0.1123
\end{tabular}

$\mathrm{RVI}$, reactor pressure vessel internal.

a) Relative error $=\frac{\text { Standard deviation }}{\text { Mean }}$.

dose rate limit sufficiently.

For RVI components, two types of casks are considered such as the cylinder type for from RVI-01 to RVI-08 and the 
Table 11. Dose Rate at Various Positions for the Box-Type Cask for RVI Components

\begin{tabular}{lcc}
\hline & Dose rate $(\mathrm{mSv} / \mathrm{hr})$ & Relative error $\left.^{2}\right)$ \\
\hline Surface & $9.56 \times 10^{-4}$ & 0.1179 \\
$10 \mathrm{~cm}$ & $9.07 \times 10^{-4}$ & 0.1179 \\
$1 \mathrm{~m}$ & $1.51 \times 10^{-4}$ & 0.0858 \\
$2 \mathrm{~m}$ & $5.99 \times 10^{-5}$ & 0.0857 \\
\hline
\end{tabular}

$\mathrm{RVI}$, reactor pressure vessel internal.

a) Relative error $=\frac{\text { Standard deviation }}{\text { Mean }}$.

Table 12. Surface Dose Rates for RV Casks

\begin{tabular}{|c|c|c|c|c|}
\hline \multirow{2}{*}{ Cask ID } & \multicolumn{4}{|c|}{ Dose rate (mSv/hr) } \\
\hline & Surface & $10 \mathrm{~cm}$ & $1 \mathrm{~m}$ & $2 m$ \\
\hline RV-01 & $2.06 \times 10^{-2}$ & $1.41 \times 10^{-2}$ & $2.40 \times 10^{-3}$ & $8.97 \times 10^{-4}$ \\
\hline RV-02 & $2.19 \times 10^{-2}$ & $1.50 \times 10^{-2}$ & $2.55 \times 10^{-3}$ & $9.53 \times 10^{-4}$ \\
\hline RV-03 & $2.11 \times 10^{-2}$ & $1.44 \times 10^{-2}$ & $2.46 \times 10^{-3}$ & $9.17 \times 10^{-4}$ \\
\hline RV-04 & $1.47 \times 10^{-2}$ & $1.01 \times 10^{-2}$ & $1.71 \times 10^{-3}$ & $6.39 \times 10^{-4}$ \\
\hline RV-05 & $1.55 \times 10^{-2}$ & $1.06 \times 10^{-2}$ & $1.80 \times 10^{-3}$ & $6.73 \times 10^{-4}$ \\
\hline RV-06 & $1.57 \times 10^{-2}$ & $1.08 \times 10^{-2}$ & $1.83 \times 10^{-3}$ & $6.84 \times 10^{-4}$ \\
\hline RV-07 & $1.15 \times 10^{-2}$ & $7.90 \times 10^{-3}$ & $1.34 \times 10^{-3}$ & $5.02 \times 10^{-4}$ \\
\hline RV-08 & $2.21 \times 10^{-2}$ & $1.52 \times 10^{-2}$ & $2.58 \times 10^{-3}$ & $9.62 \times 10^{-4}$ \\
\hline RV-09 & $2.04 \times 10^{-2}$ & $1.40 \times 10^{-2}$ & $2.37 \times 10^{-3}$ & $8.87 \times 10^{-4}$ \\
\hline RV-10 & $1.52 \times 10^{-2}$ & $1.04 \times 10^{-2}$ & $1.77 \times 10^{-3}$ & $6.61 \times 10^{-4}$ \\
\hline $\mathrm{RV}-11$ & $1.39 \times 10^{-2}$ & $9.50 \times 10^{-3}$ & $1.62 \times 10^{-3}$ & $6.03 \times 10^{-4}$ \\
\hline $\mathrm{RV}-12$ & $1.39 \times 10^{-2}$ & $9.50 \times 10^{-3}$ & $1.62 \times 10^{-3}$ & $6.03 \times 10^{-4}$ \\
\hline RV-13 & $2.21 \times 10^{-2}$ & $1.52 \times 10^{-2}$ & $2.58 \times 10^{-3}$ & $9.62 \times 10^{-4}$ \\
\hline $\mathrm{RV}-14$ & $2.21 \times 10^{-2}$ & $1.52 \times 10^{-2}$ & $2.58 \times 10^{-3}$ & $9.62 \times 10^{-4}$ \\
\hline $\mathrm{RV}-15$ & $6.97 \times 10^{-1}$ & $4.78 \times 10^{-1}$ & $8.12 \times 10^{-2}$ & $3.03 \times 10^{-2}$ \\
\hline $\mathrm{RV}-16$ & $6.97 \times 10^{-1}$ & $4.78 \times 10^{-1}$ & $8.12 \times 10^{-2}$ & $3.03 \times 10^{-2}$ \\
\hline RV-17 & $4.77 \times 10^{-1}$ & $3.27 \times 10^{-1}$ & $5.56 \times 10^{-2}$ & $2.08 \times 10^{-2}$ \\
\hline RV-18 & $2.83 \times 10^{-1}$ & $1.94 \times 10^{-1}$ & $3.29 \times 10^{-2}$ & $1.23 \times 10^{-2}$ \\
\hline $\mathrm{RV}-19$ & $5.19 \times 10^{-1}$ & $3.55 \times 10^{-1}$ & $6.04 \times 10^{-2}$ & $2.26 \times 10^{-2}$ \\
\hline RV-20 & $1.32 \times 10^{-4}$ & $9.07 \times 10^{-5}$ & $1.54 \times 10^{-5}$ & $5.76 \times 10^{-6}$ \\
\hline $\mathrm{RV}-21$ & $2.80 \times 10^{-1}$ & $1.92 \times 10^{-1}$ & $3.26 \times 10^{-2}$ & $1.22 \times 10^{-2}$ \\
\hline RV-22 & $2.80 \times 10^{-1}$ & $1.92 \times 10^{-1}$ & $3.26 \times 10^{-2}$ & $1.22 \times 10^{-2}$ \\
\hline$R V-23$ & $2.98 \times 10^{-1}$ & $2.04 \times 10^{-1}$ & $3.48 \times 10^{-2}$ & $1.30 \times 10^{-2}$ \\
\hline RV-24 & $1.51 \times 10^{-2}$ & $1.03 \times 10^{-2}$ & $1.76 \times 10^{-3}$ & $6.57 \times 10^{-4}$ \\
\hline $\mathrm{RV}-25$ & $8.59 \times 10^{-5}$ & $5.89 \times 10^{-5}$ & $1.00 \times 10^{-5}$ & $3.74 \times 10^{-6}$ \\
\hline RV-26 & $3.20 \times 10^{-3}$ & $2.19 \times 10^{-3}$ & $3.72 \times 10^{-4}$ & $1.39 \times 10^{-4}$ \\
\hline RV-27 & $3.02 \times 10^{-3}$ & $2.07 \times 10^{-3}$ & $3.52 \times 10^{-4}$ & $1.31 \times 10^{-4}$ \\
\hline RV-28 & $2.88 \times 10^{-3}$ & $1.97 \times 10^{-3}$ & $3.35 \times 10^{-4}$ & $1.25 \times 10^{-4}$ \\
\hline $\mathrm{RV}-29$ & $2.52 \times 10^{-3}$ & $1.73 \times 10^{-3}$ & $2.94 \times 10^{-4}$ & $1.10 \times 10^{-4}$ \\
\hline RV-30 & $8.43 \times 10^{-4}$ & $5.77 \times 10^{-4}$ & $9.82 \times 10^{-5}$ & $3.67 \times 10^{-5}$ \\
\hline RV-31 & $5.99 \times 10^{-3}$ & $4.10 \times 10^{-3}$ & $6.97 \times 10^{-4}$ & $2.60 \times 10^{-4}$ \\
\hline
\end{tabular}

$\mathrm{RV}$, reactor pressure vessel.

box type for from RVI-09 to RVI-19. Fig. 9 shows the cylindertype cask modeling and Table 10 provides surface dose rates for the cylinder-type cask when the photon intensity of $7.4 \times$ $10^{13}$ photons/s/MeV is assumed for RVI components. And Table 11 provides surface dose rates for the box-type cask when the photon intensity of $3.0 \times 10^{11}$ photons $/ \mathrm{s} / \mathrm{MeV}$ is as-
Table 13. Surface Dose Rates for RVI Casks

\begin{tabular}{lcccc}
\hline \multirow{2}{*}{ Cask ID } & \multicolumn{4}{c}{ Dose rate (mSV/hr) } \\
\cline { 2 - 5 } & Surface & $10 \mathrm{~cm}$ & $1 \mathrm{~m}$ & $2 \mathrm{~m}$ \\
\hline RVI-01 & $9.11 \times 10^{-4}$ & $7.38 \times 10^{-4}$ & $2.51 \times 10^{-4}$ & $1.17 \times 10^{-4}$ \\
RVI-02 & $8.10 \times 10^{-3}$ & $6.56 \times 10^{-3}$ & $2.24 \times 10^{-3}$ & $1.04 \times 10^{-3}$ \\
RVI-03 & $9.48 \times 10^{-4}$ & $7.68 \times 10^{-4}$ & $2.62 \times 10^{-4}$ & $1.21 \times 10^{-4}$ \\
RVI-04 & $1.55 \times 10^{-2}$ & $1.26 \times 10^{-2}$ & $4.29 \times 10^{-3}$ & $1.99 \times 10^{-3}$ \\
RVI-05 & $9.61 \times 10^{-4}$ & $7.78 \times 10^{-4}$ & $2.65 \times 10^{-4}$ & $1.23 \times 10^{-4}$ \\
RVI-06 & $1.62 \times 10^{-3}$ & $1.32 \times 10^{-3}$ & $4.48 \times 10^{-4}$ & $1.23 \times 10^{-4}$ \\
RVI-07 & 1.66 & 1.58 & $2.62 \times 10^{-1}$ & $1.04 \times 10^{-1}$ \\
RVI-08 & $7.09 \times 10^{-1}$ & $6.73 \times 10^{-1}$ & $1.12 \times 10^{-1}$ & $4.44 \times 10^{-2}$ \\
RVI-09 & $6.27 \times 10^{-4}$ & $5.94 \times 10^{-4}$ & $9.90 \times 10^{-5}$ & $3.93 \times 10^{-5}$ \\
RVI-10 & $5.90 \times 10^{-4}$ & $5.60 \times 10^{-4}$ & $9.32 \times 10^{-5}$ & $3.70 \times 10^{-5}$ \\
RVI-11 & $6.63 \times 10^{-4}$ & $6.29 \times 10^{-4}$ & $1.05 \times 10^{-4}$ & $4.15 \times 10^{-5}$ \\
RVI-12 & $5.81 \times 10^{-4}$ & $5.51 \times 10^{-4}$ & $9.17 \times 10^{-5}$ & $3.64 \times 10^{-5}$ \\
RVI-13 & $2.05 \times 10^{-5}$ & $1.94 \times 10^{-5}$ & $3.24 \times 10^{-6}$ & $1.28 \times 10^{-6}$ \\
RVI-14 & $1.78 \times 10^{-5}$ & $1.68 \times 10^{-5}$ & $2.80 \times 10^{-6}$ & $1.11 \times 10^{-6}$ \\
RVI-15 & $1.16 \times 10^{-3}$ & $1.10 \times 10^{-3}$ & $1.84 \times 10^{-4}$ & $7.28 \times 10^{-5}$ \\
RVI-16 & $1.32 \times 10^{-2}$ & $1.25 \times 10^{-2}$ & $2.08 \times 10^{-3}$ & $8.26 \times 10^{-4}$ \\
RVI-17 & $6.17 \times 10^{-4}$ & $5.86 \times 10^{-4}$ & $9.75 \times 10^{-5}$ & $3.87 \times 10^{-5}$ \\
RVI-18 & $7.51 \times 10^{-4}$ & $7.12 \times 10^{-4}$ & $1.19 \times 10^{-4}$ & $4.70 \times 10^{-5}$ \\
RVI-19 & $8.21 \times 10^{-4}$ & $7.79 \times 10^{-4}$ & $1.30 \times 10^{-4}$ & $5.14 \times 10^{-5}$ \\
\hline RVI1 & & & &
\end{tabular}

$\mathrm{RVI}$, reactor pressure vessel internal.

sumed for RVI components.

Tables 12 and 13 provide the surface dose rate of various casks containing RV and RVI components, respectively.

From Table 12, the dose rates of various casks for RV components distributed much lower than the limit. Especially, RV-15 and RV-16 provide the highest surface dose rates due to their highest source intensities. From Table 13, the dose rates of various casks for RVI components are also satisfactory. The highest dose rate happens in the RVI-07 cask due to its much higher source intensity. It needs to be adjusted contents of such a cask which carries highly radioactive components in order to decrease thickness of the box-type cask and to reduce transport burden in the future.

\section{Conclusion}

In this paper, various cut-off pieces from RV and RVI components are characterized to transport safely by using boxand cylinder-type specialized casks. The radiation source intensities are obtained from activation analysis using ORIGEN-S calculation and surface dose rates for various casks are also calculated from MCNP simulations. Optimized cask loading strategies should be prepared using activation calculation for activated components, cutting plan on each position separated by radioactive waste level, radiation intensity 
and weight on each cut-off piece and cask size. From the calculation results for total 50 casks, it is found that the strategy is proposed adequately by satisfying both load weight and radiation dose rate requirement on the surface of cask. However, it might be better for economical transportation by loading higher radioactive contents and the heavier components. As a conclusion, this works shall contribute to estimate optimal loading plan of RV and RVI components for the decommissioning nuclear power plants, and the results can be used to prepare radioactive wastes-ILW, LLW, VLLW-disposal plan.

\section{Conflict of Interest}

No potential conflict of interest relevant to this article was reported.

\section{Acknowledgements}

This research was supported by a grant from Energy Technology Development Program Funded by Ministry of Trade, Industry and Energy of Korean government (No. 20161510300430).

\section{Author Contribution}

Conceptualization: Park K, Kim H, Kim N, Lee C, Lee Y, Lee J, Hwang Y, Lee M, Lee D, Jung D. Data curation: Park K, Kim H, Kim N, Lee C, Lee Y, Lee D, Jung D. Methodology: Sohn H. Project administration: Park K, Kim N, Lee C, Lee Y, Lee J, Hwang Y, Lee M, Lee D, Jung D. Visualization: Sohn H.
Writing - original draft: Sohn H. Writing - review \& editing: Park K, Kim H. Resources: Kim H, Sohn H. Software: Sohn H. Supervision: Lee J, Hwang Y, Lee M.

\section{References}

1. Mostecak A, Bedekovic G. Metal waste management and recycling methods in the nuclear power plant decommissioning and dismantling process. Rudarsko-geolosko-naftni zbornik. 2018;33:25-33.

2. Hansson T, Norberg T, Knutsson A, Fors P, Sandebert C. An assessment of the decommissioning cost for the Ringhals site. Stockholm, Sweden; Swedish Nuclear Fuel and Waste Management Co.; 2013.

3. National Education Association. Recycling and reuse of materials arising from the decommissioning of nuclear facilities. Paris, France: OECD Publishing; 2017.

4. Sohn HD, Park KS, Kim HW, Kim CW, Hwang YH, Yoon JY. The study for activation evaluation on activated structures in nuclear power plant with 40 years operation history. Ann Nucl Energy. 2020;141:107305.

5. Pelowitz DB. MCNP6 user's manual - code version 6.1 (LA-CP13-00634). Los Alamos, NM: Los Alamos National Laboratory; 2013.

6. Gauld IC. ORIGEN-S: depletion module to calculate neutron activation, actinide transmutation, fission product generation, and radiation source terms (ORNL/TM-2005/39 version 6.1). Oak Ridge, TN: Oak Ridge National Laboratory; 2011.

7. Evans JC, Lepel EL, Sanders RW, Wilkerson CL, Silker W, Thomas CW, et al. Long-lived activation products in reactor materials (No. NUREG/CR-3474; PNL-4824). Richland, WA: Pacific Northwest Lab.; 1984 